\title{
Effect of Exercise Position on Percent Changes in Serratus Anterior Muscle Thickness
}

\author{
Joseph Day* \\ University of South Alabama
}

Received: October 20, 2016; Accepted: November16, 2016; Published: January 17, 2017

*Corresponding author: Dr Joseph Day, University of South Alabama Address : HAHN 20115721 USA Drive North, Mobile, AL 36688-0002, Fax 251-445-9238,Tel : 937-231-8231,E-mail : josephday@southalabama.edu

\begin{abstract}
Objective: A reliable method for measuring SA thickness with ultrasound imaging has been reported, yet the ability to detect differences in levels of contractility has yet to be established. The purpose of this study was to determine if there are differences in the percent change in thickness of the SA during four different exercise positions performed by healthy individuals.

Design: Observational study

Setting: Laboratory

Participants: Twenty-eight healthy participants, mean age 26 (SD 4, 57\% female, $100 \%$ right handed)

Methods: Volunteers were positioned in a standardized sitting posture and anatomical landmarks were marked for ultrasound probe positioning. Two resting and two contractile ultrasound images were taken for each exercise position and the average thickness between trials was used in the data analysis. The exercise positions included sitting scaption to $90^{\circ}$, sitting scaption to 120

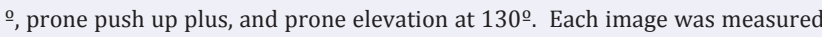
with on-screen calipers. Descriptive statistics were run for all participants. A repeated measures Friedman analysis was used to determine differences in the percent change in thickness between each exercise position.
\end{abstract}

Main Outcome Measure: Percent change in serratus anterior muscle thickness from rest to contraction using musculoskeletal ultrasound imaging.

Results: There were no significant differences between the percent changes in muscle thickness when comparing 4 different exercise positions ( $p$ $=.624$ ).

Conclusions: Diagnostic ultrasound imaging does not appear to be sensitive enough to detect differences in SA percent change in thickness between exercise positions in healthy individuals.

Using this methodology, the percent change in SA thickness from rest to contraction, regardless of position, is expected to be approximately 30 to $40 \%$ in healthy young individuals.

Keywords: SA thickness, exercise position, healthy subjects

\section{Introduction}

The serratus anterior (SA) is a key muscle of the scapula humeral joint functioning in both stabilization and mobilization of the scapula [1-4]. Because of its important role as a dynamic scapular stabilizer, several assessment tools have been used to investigate SA muscle performance $[5,6]$. Unfortunately, these assessment tools have limitations. Techniques used to manual muscle test the SA are easy to perform but do not appear to isolate the SA [5]. ElectroMyographic Activity (EMG) and motion analysis provide researchers with isolated and detailed information but interpretation is difficult $[7,8]$. Unlike other assessment tools, Ultrasound Imaging (UI) provides isolated real time visualization of muscle contraction, thus allowing researchers and clinicians quick interpretation of the contraction of a specific muscle $[9,10]$. UI has been shown to be a valid and reliable assessment tool for measuring thickness and cross sectional areas of the transversus abdominus, obliques [11], lumbar multifidi [12], quadriceps [13], lower trapezius [14], and cervical musculature [15]; yet there is a limited body of evidence related to the psychometric properties of measuring SA thickness with UI.

A few studies have investigated muscle thickness measures of the SA in both healthy [16-18] and pathological populations [19]. In general, between and within day intra-rater reliability measuring SA thickness has been shown to be good to excellent in health individuals positioned in glenohumeral scaption [16, 17]. Only one study has investigated inter rater reliability of SA thickness and the results were modest to poor [17].

Other studies have investigated the ability to detect changes in SA thickness using UI. Two studies found no differences in healthy individuals when adding various loads to the UE in scaption at both 90 degrees [16], and 120 degrees of elevation [18]. However, increasing loads during upper extremity elevation does not appears to effect EMG activity of the serratus anterior [20] and therefore, may explain why no changes in the SA thickness were observed between loads using diagnostic ultrasound imaging. One other study has investigated SA muscle thickness in patients with LE compared to controls. Preliminarily and with a small sample size, it appears that UI was able to detect significant differences in the change of muscle thickness from rest to contraction when comparing patients to controls but the mean differences were not beyond minimal detectable change values [16]. Another manuscript by Seitz et al, reported no significant differences in SA muscle thickness in health individuals with and without observable scapular dyskinesia [21].

With the available evidence in UI assessment of the SA, it 
would appear that the sensitivity of UI to detect difference in SA muscle thickness is still unknown. The literature demonstrates that change in body position has resulted in an increase in muscle thickness of the internal obliques and transversus abdominus in healthy individuals [22]. In addition, it is well established that difference in SA EMG activity is easily distinguishable when changing the position or weight bearing status of the shoulder [23-25]. Therefore, it is reasonable to assume that shoulder exercises that vary in position may produce differences in UI measured muscle thickness of the SA. The purpose of this study is to investigate the ability of UI to detect differences in muscle thickness between 4 scapulo humeral exercises in healthy individuals. Measurable differences in muscle thickness between scapulo humeral exercises will provide evidence for the sensitivity and use of UI in evaluating the contractility of the SA.

\section{Methods}

Healthy males and females between the ages of 19-35 years old were included in the study. Participants were excluded for reporting previous injuries or surgeries to the trunk and/or upper extremities within the last year.

Height, weight, gender, occupation, age, and activity levels were collected from each participant at the beginning of each session. The participant's activity level was based on a specific activities questionnaire by Brophy et al. [27] with a maximum score of 20, indicating a very high level of daily upper extremity function, and a minimum of 4 , indicating the participant "Never or less than once a month" performed specific functional activities with their upper extremities.

\section{Muscle Identification}

Participants were asked to sit on a backless chair. To control sitting posture during the procedure, participants were asked to sit up straight and then perform trunk flexion and extension three times to find a neutral seated position [26]. The participant's dominant arm was identified prior to testing by asking the individual "Which arm would you use to throw a ball?" One of the investigators resisted the participant's shoulder extension so that the border of the latissimus dorsi could be identified. Next, a pen mark was made on the participants mid axillary line between the pectoralis major and the latissimus dorsi at the rib that corresponded to the inferior angle of the scapula [21].

\section{Procedures}

In B mode, A Z6 MSK Mindray Ultrasound Scanner with a $40 \mathrm{~mm}$ linear transducer was used to collect the data. For each participant, data was collected in four different positions, sitting scaption to $90^{\circ}$, sitting scaption to $120^{\circ}$, prone horizontal abduction to $130^{\circ}$, and push up plus. The authors chose the aforementioned exercises because of the large reported differences in EMG activity of the SA among the four positions [23-25].The order of testing was counter balanced to avoid effects of fatigue. For all trials, the same investigator monitored arm position and trunk posture.

Scaption to $90^{\circ}$ and $120^{\circ}$ : With the participant seated in a neutral spine posture, the Upper Extremity (UE) was passively positioned on an adjustable table to $75^{\circ}$ of elevation. The participants were then positioned in scaption, defined as $30^{\circ}$ anterior to the frontal plane, with the UE resting on the table. The ultrasound transducer was positioned vertically over the pen marker while the first resting baseline ultrasound image was taken. The participants were then instructed to raise their arm to $90^{\circ}$ or $120^{\circ}$ (Figures 1 and 2). A second ultrasound image was then taken within 5 seconds of reaching the apex of movement. Each participant was provided a visual goal and a tactile response when the participant had achieved their goal of UE elevation.

Push up plus: The participants were instructed to lie prone with their chest supported on an adjustable pediatric bench. With the trunk supported by the bench, the hips were slightly flexed, knees extended, and feet contacting the floor. The upper extremities were supported on either side of the bench by placing a short stool and pillow under the participant's upper extremities with the shoulder placed in $90^{\circ}$ abduction, neutral shoulder

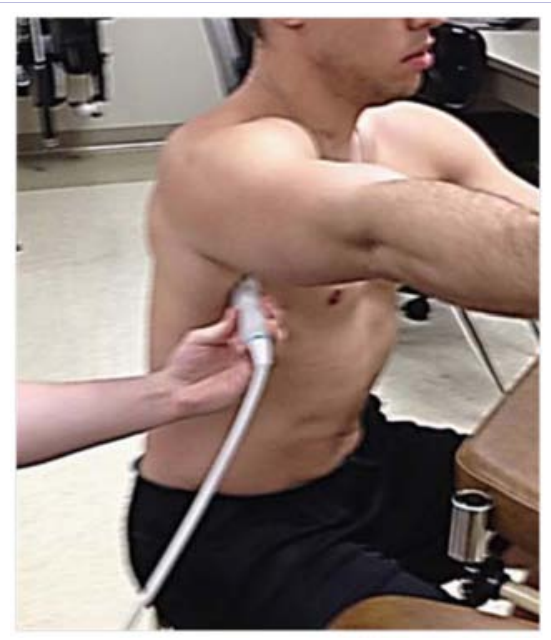

Figure 1: Sitting Scaption to $90^{\circ}$

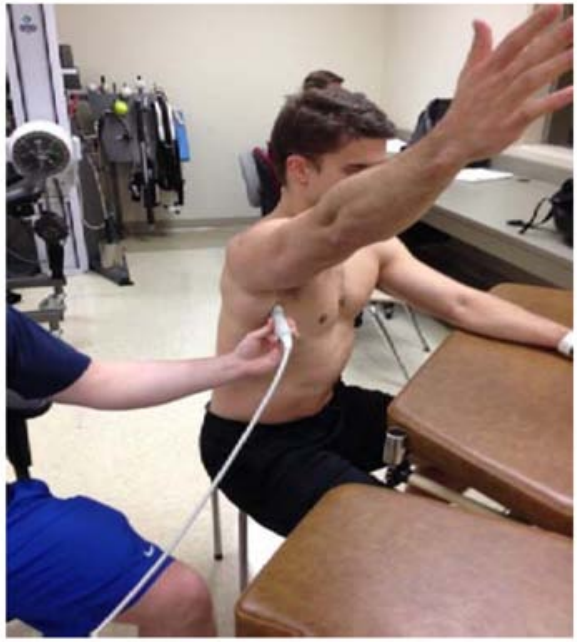

Figure 2: Sitting Scaption to $120^{\circ}$ 
rotation, and elbow flexed. Support through the UE was provided in order to prevent weight bearing through the hand during the resting data collection. Weight bearing through the hand has been shown to produce moderate SA activity [27]. The ultrasound transducer was then placed on the predetermined reference point and the first resting image was taken. The support for the upper extremities was removed, and participants were asked to press their hands into the floor, extend the elbows, and protract the scapula. The second ultrasound picture was taken within 5 seconds of completing the contraction (Figure 3).

Prone horizontal abduction to $130^{\circ}$ (Prone Y): Finally, the participants were position prone on a treatment table with the cervical spine in neutral. The participant's shoulder was then abducted to $130^{\circ}$, elbow flexed to 45 and the entire UE was placed in a resting position on a chair with a pillow. The ultrasound transducer was then placed on the predetermined reference point and the first resting baseline ultrasound was taken. For contractile images, participants were asked to raise the UE to a level that was even with the participant's trunk while the elbow was extended and the thumb pointed upward (Figure 4).

For all test positions, a second trial was performed allowing for a total of two resting and two contractile images. The participants were allocated 30 seconds rest between trials for each test position.

\section{Data Synthesis}

After data collection, on screen calipers were used to measure SA muscle thickness by a single investigator. The investigator measuring muscle thickness was trained by the primary investigator whom is certified and has research experience with diagnostic ultrasound imaging. To ensure inter rater reliability, the investigator measuring on screen SA muscle thickness for this study and the primary investigator randomly chose 1 patient and independently measured the absolute thickness across all conditions (rest and contraction at 90 degrees elevation, 120 degrees elevation, quadruped push up plus, and prone Y).

Five vertical measurements equally spaced apart and spanning the width of the rib, were taken from the anterior surface of the rib to the superior facial border of the SA (Figures 5 and 6). Using these five measurements, an average was taken

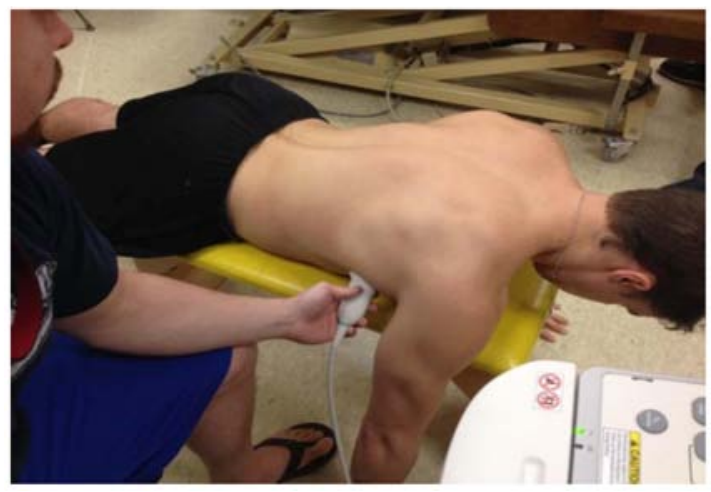

Figure 3: Push up Plus

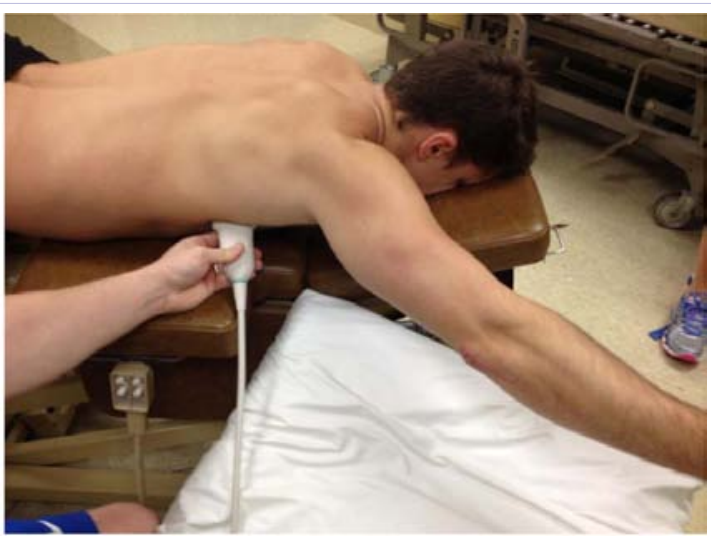

Figure 4: Prone Horizontal Abduction to $130^{\circ}$

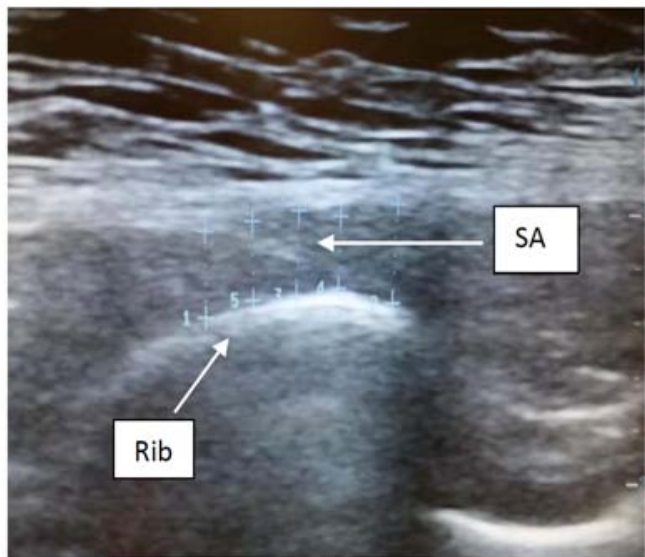

Figure 5: Resting Thickness Measure of the Serratus Anterior The rib was used as a reference for measurement of the serratus anterior (SA). Five vertical perforated yellow lines, spaced out to encompass the width of the rib, were drawn from the rib to the superior fascial border of the SA. The average of the five measurements was used to represent SA thickness.

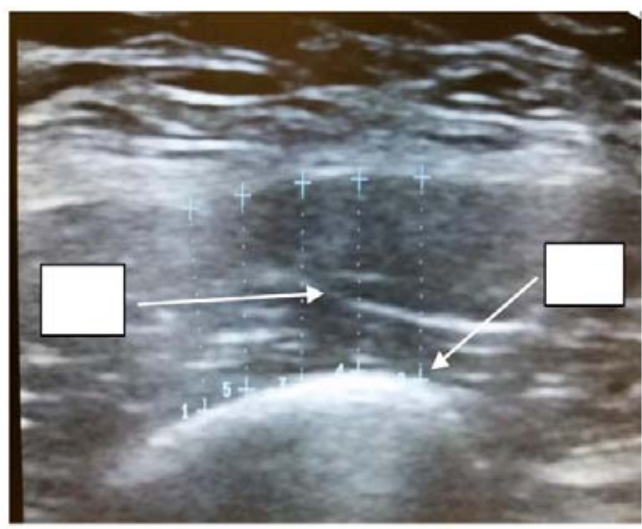

Figure 6: Contractile Thickness Measure of the Serratus Anterior The rib was used as a reference for measurement of the serratus anterior (SA). Five vertical perforated yellow lines, spaced out to encompass the width of the rib, were drawn from the rib to the superior fascial border of the SA. The average of the five measurements was used to represent SA thickness. 
to represent SA thickness [21]. The two measures of resting thickness and two measure of contractile thickness were both averaged together for every individual in each position. Percent change in thickness was then calculated using the following formula for each individual in each position.

((Contractile thickness - resting thickness)/Resting thickness) $* 100 \%$

\section{Statistical Analysis}

An intra class correlation coefficient (ICC ${ }^{3,2}$ ) was used to measure the on screen measures of SA muscle thicknesses across the conditions previously chosen by the examiners. Measures of central tendency and variability were calculated for each participant's, Body Mass Index (BMI), weight, height, and shoulder activity level. Test for sphericity (Mauchley's) and normality (Shapiro-Wilk) on the percent change in muscle thickness indicated a nonparametric distribution of data. We therefore chose a repeated measure Friedman's design to analyze potential differences in the percent change in thickness between each of the 4 exercise positions.

\section{Results}

Data was collected on 30 participants, however two participants were excluded secondary to poor imaging quality. Therefore, we included 28 participants (mean age 26 (SD 4, 57\% female, $100 \%$ right handed) in our statistical analysis. The interrater reliability for on screen measurements of the SA obtained through UI was excellent $\left(\mathrm{ICC}^{3,2}=.93\right)$.Participants' activity levels were variable, ranging from 3-17, while participants' mean BMI was considered to be in the overweight range for adults over the age of 20 [28] (Table 1). Friedman's repeated measures comparison revealed no significant differences between positions $(p=.624)$. There was a consistent change in mean thickness, from rest to contraction, of the SA across all positions of at least $30 \%$, illustrated in Figure 7.

\section{Discussion}

Significant differences in the percent change of SA thickness were not detected between exercise positions in healthy individuals, but our procedures were sensitive enough to detect $30 \%$ to $40 \%$ increases between rest and contraction of the SA across all exercise positions. This percent increase in thickness is similar to previous studies on healthy individuals $[16-19,21]$. Therefore, the consistency in the percent change in

\section{Table 1: Participant Characteristics}

\begin{tabular}{|c|c|c|c|c|}
\hline & Minimum & Maximum & Mean & $\begin{array}{c}\text { Std. } \\
\text { Deviation }\end{array}$ \\
\hline BMI * & 23.05 & 29.82 & 26.28 & 3.01 \\
\hline Weight (kg) & 56.70 & 108.86 & 73.55 & 15.03 \\
\hline Height (m) & 1.57 & 1.91 & 1.74 & 0.10 \\
\hline $\begin{array}{c}\text { Activity } \\
\text { Total } \neq\end{array}$ & 3.00 & 17.00 & 11.41 & 3.51 \\
\hline
\end{tabular}

${ }^{*}$ BMI calculated using $\left(\mathrm{WT}_{\mathrm{kg}} / \mathrm{HT}_{\mathrm{m}}{ }_{\mathrm{m}}\right) \neq$ Measurements were taken based on specific activities questionnaire. Max: 20, Min: 0 (Brophy et al, 2005).

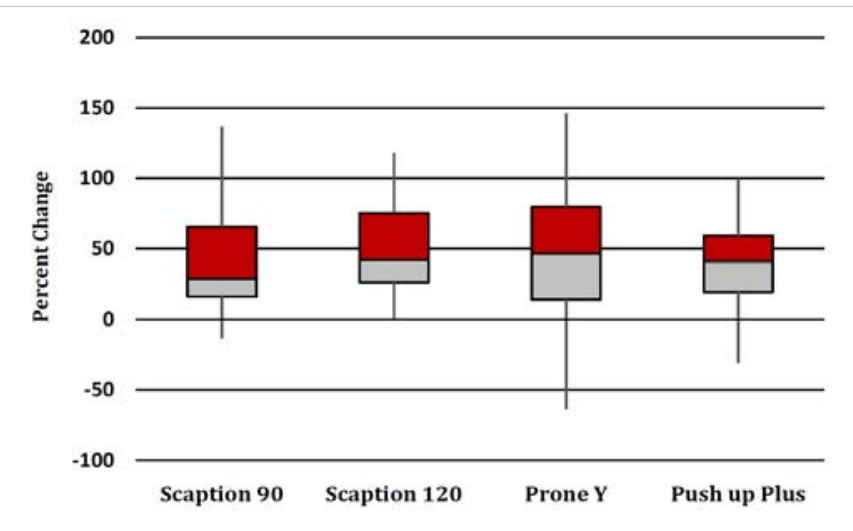

Figure 7: Percent Change in Serratus Anterior Thickness

muscle thickness measures of the SA in our study and previous studies provides valuable normative reference data for future studies.

The inability to detect differences in the percent change in SA muscle thicknesses across exercises in healthy individuals is also consistent with previous literature. Seitz et al, recently determined that there were no detectable differences in the percent change in muscle thickness between healthy individuals observed to have scapular dyskinesia and healthy individuals with no observable dyskinesia [21]. Talbot and Witt found no differences in absolute muscle thickness of the SA between active arm elevation and loaded elevation at $120^{\circ}$ [17]. Similarly, Day and Uhl found no significant differences in absolute muscle thickness when loading the upper extremity with various weights in the same position [16].

Despite the lack of sensitivity found in healthy individuals, the authors propose that the results of comparing a pathological population to a control population might be different. In support of this hypothesis, a recent study compared SA muscle thickness of patient's with LE to matched controls. The authors in this study found an average of 35\% increase in thickness for matched controls and an approximate $17 \%$ increase in muscle thickness for patients with LE [19]. In the quest of determining the sensitivity of the measuring SA thickness with UI, future research should examine the same methodology on patients with shoulder pathologies and compare the results to a control group.

It is also imperative to consider the limitations to our methodology when interpreting the findings of this study. For each position, the transducer was placed at the level of the inferior angle of the scapula, corresponding to the lower portion of the $\mathrm{SA}$. It has been hypothesized that the primary action of the lower portion of SA is upward rotation while the upper portion of the SA primarily protracts the scapula [29]. Because the transducer was placed on the inferior portion of the SA muscle and the push up plus results in scapular protraction, the change in thickness recorded at the lower portion of the SA for the push-up plus may not have been accurate. Therefore, future research should consider both the placement of the transducer and whether the primary intent of the exercise is scapular upward rotation or protraction. 
Our procedures were designed to capture changes in the width of a muscle two dimensionally. Because muscles contraction occurs three dimensionally, changes in length and height may account for unseen changes in the SA between the exercise positions [30]. In addition, the $40 \mathrm{~mm}$ linear transducer depicts a localized area of the SA muscle that envelopes a broad area of the lateral rib cage. One method of overcoming the fore mentioned limitations is to calculate changes in the Cross Sectional Area (CSA) of the SA [31-33]. However, the authors chose not to explore CSA calculations secondary to the complexity of the measurements rendering the procedure less viable for clinical utility [30].

Another important concept to consider when interpreting our results is that compensatory muscle recruitment patterns may have occurred. First, no participants received feedback for SA muscle activation during the exercises. A recent EMG study has demonstrated that muscle recruitment is more consistent when multimodal instruction and feedback is given [34]. Second, because multiple scapula humeral muscles are activated during the four selected exercises [35], it is possible that some participants were able to perform the exercises by engaging other scapula humeral muscles while minimally contracting the SA. To that end, a recent morphological study of the SA may also support our findings. In a study on rat models, the rostral SA is composed of approximately 85\% type 2 fast fatiguing muscle fibers [36]. Because the SA is easily fatigable, it is likely that some participants recruited more of the upper trapezius, a slow fatiguing muscle, during arm elevation [37]. In order to ensure proper and consistent SA contraction, future studies should consider giving each patient verbal and tactile feedback and limiting the number of contractile repetitions during studies.

Finally, our calculated post hoc power was low secondary to the high variability in the percent change in muscle thicknesses. Given the variability in this study, we would need 93 participants for percent change of $61 \%$ and $44 \%$ and SD of $56 \%$. Higher variability might be expected in this study secondary to the utilization of 2 different mean values in our percentage change equation. Compared to other similar studies, the variability reported in this study is comparable [21]. The consistency of the method used in this study can be improved by using verbal cues for proper contraction and ensuring that the timing of the captured image is more consistent. Improved consistency will reduce the variability and lower the amount of participants needed to see a significant change if a difference really exists.

\section{Conclusion}

Diagnostic Ultrasound does not appear to be sensitive enough to detect differences in thickness of the SA in different exercise positions with healthy young individuals. Ultrasound imaging generally demonstrates increases in thickness of the SA from a state of rest to a contracting position. This change was approximately 30\% across all conditions; however the large degree of variability should be noted. Future research is needed to confirm the clinical utility of this procedure with a pathological population.

\section{References}

1. Voight ML, Thomson BC. The role of the scapula in the rehabilitation of shoulder injuries. J Athl Train. 2000;35(3):364-372.

2. Lucado AM. Scapular Muscle Imbalance: Implications for Shoulder Pain and Pathology. Physical Therapy Reviews. 2011;16(5):356-364. doi.org/10.1179/1743288X11Y.0000000039.

3. Hidetomo S, K A Swanik, K C Huxel, J D Kelly IV, Swanik CB. Alterations in Upper Extremity Motion after Scapular-Muscle Fatigue. Journal of Sport Rehabilitation. 2006;15:71-88.

4. Kibler W. The role of the scapula in athletic shoulder function. American Journal of Sports Medicine. 1998;26(2)325-337.

5. Michener LA, Boardman ND, Pidcoe PE, Frith AM. Scapular muscle tests in subjects with shoulder pain and functional loss: reliability and construct validity. Phys Ther. 2005;85(11):1128-1138.

6. Struyf F, Nijs J, Mottram S, Roussel NA, Cools AM, Meeusen R. Clinical assessment of the scapula: a review of the literature. Br J Sports Med. 2014;48(11):883-890. doi: 10.1136/bjsports-2012-091059.

7. Hackett L, Reed D, Halaki M, Ginn KA. Assessing the validity of surface electromyography for recording muscle activation patterns from serratus anterior. J Electromyogr Kinesiol. 2014;24(2):221-227. doi: 10.1016/j.jelekin.2014.01.007.

8. Ludewig PM, Phadke V, Braman JP, Hassett DR, Cieminski CJ, LaPrade RF. Motion of the shoulder complex during multiplanar humeral elevation. J Bone Joint Surg Am. 2009;91(2):378-389. doi: 10.2106/ JBJS.G.01483.

9. Teyhen DS. "Rehabilitative ultrasound imaging for assessment and treatment of musculoskeletal conditions". Man Ther. 2011;16(1):4445. doi: 10.1016/j.math.2010.06.012.

10. Whittaker JL, Stokes M. Ultrasound imaging and muscle function. J Orthop Sports Phys Ther. 2011;41(8):572-580. doi: 10.2519/ jospt.2011.3682.

11. Costa LO, Maher CG, Latimer J, Smeets RJ. Reproducibility of rehabilitative ultrasound imaging for the measurement of abdominal muscle activity: a systematic review. Phys Ther. 2009;89(8):756-769. doi: $10.2522 /$ ptj.20080331.

12. Kiesel KB, Uhl TL, Underwood FB, Rodd DW, Nitz AJ. Measurement of lumbar multifidus muscle contraction with rehabilitative ultrasound imaging. Man Ther. 2007;12(2):161-166. doi:10.1016/j. math.2006.06.011.

13. Freilich RJ, Kirsner RL, Byrne E. Isometric strength and thickness relationships in human quadriceps muscle. Neuromuscul Disord. 1995;5(5):415-422.

14. O'Sullivan C, Bentman S, Bennett K, Stokes M. Rehabilitative ultrasound imaging of the lower trapezius muscle: technical description and reliability. J Orthop Sports Phys Ther. 2007;37(10):620-626. doi:10.2519/jospt.2007.2446.

15. Ishida H, Suehiro T, Kurozumi C, Ono K, Watanabe S. Ultrasound imaging of the diagonal dimension of the deep cervical flexor muscles: A reliability study on healthy subjects. J Bodyw Mov Ther. 2015;19(3):417-420. doi: 10.1016/j.jbmt.2014.09.004.

16. Day JM, Uhl T. Thickness of the lower trapezius and serratus anterior using ultrasound imaging during a repeated arm lifting task. Man Ther. 2013;18(6):588-593. doi: 10.1016/j.math.2013.07.003.

17. Talbott NR, Witt DW. Ultrasound imaging of the serratus anterior muscle at rest and during contraction. Clin Physiol Funct Imaging. 2013;33(3):192-200. doi: 10.1111/cpf.12012. 
18. Talbott NR, Witt DW. Ultrasound examination of the serratus anterior during scapular protraction in asymptomatic individuals: reliability and changes with contraction. PM R. 2014;6(3):227-234. doi: 10.1016/j.pmrj.2013.09.003.

19.Day JM, Bush H, Nitz AJ, Uhl TL. Scapular Muscle Performance in Individuals With Lateral Epicondylalgia. J Orthop Sports Phys Ther. 2015; 45(5):414-24. doi: 10.2519/jospt.2015.5290.

20.Wattanaprakornkul D, Halaki M, Boettcher C, Cathers I, Ginn KA. A comprehensive analysis of muscle recruitment patterns during shoulder flexion: an electromyographic study. Clin Anat 2011;24(5):619-626. doi: 10.1002/ca.21123.

21.Seitz AL, Baxter CJ, Benya K. Muscle thickness measurements of the lower trapezius with rehabilitative ultrasound imaging are confounded by scapular dyskinesis. Man Ther. 2015;20(4):558-563. doi: 10.1016/j.math.2015.01.002.

22. Sugaya T, Abe Y, Sakamoto M. Ultrasound evaluation of muscle thickness changes in the external oblique, internal oblique, and transversus abdominis muscles considering the influence of posture and muscle contraction. J Phys Ther Sci. 2014;26(9):1399-1402. doi: $10.1589 /$ jpts.26.1399.

23. Ekstrom RA, Donatelli RA, Soderberg GL. Surface electromyographic analysis of exercises for the trapezius and serratus anterior muscles. J Orthop Sports Phys Ther. 2003;33(5):247-258. doi:10.2519/ jospt.2003.33.5.247

24. Hwang UJ, Kwon OY, Jeon IC, Kim SH, Weon JH. Effect of Humeral Elevation Angle on Electromyographic Activity in the Serratus Anterior During the Push-up Plus Exercise. J Sport Rehabil. 2015;122. doi:10.1123/jsr.2015-0090.

25. Piraua AL, Pitangui AC, Silva JP, Pereira dos Passos MH, Alves de Oliveira VM, Batista Lda S, et al. Electromyographic analysis of the serratus anterior and trapezius muscles during push-ups on stable and unstable bases in subjects with scapular dyskinesis. J Electromyogr Kinesiol. 2014;24(5):675-681. doi: 10.1016/j.jelekin.2014.05.009.

26. Lynch SS, Thigpen CA, Mihalik JP, Prentice WE, Padua D. The effects of an exercise intervention on forward head and rounded shoulder postures in elite swimmers. Br J Sports Med. 2010;44(5):376-381. doi: 10.1136/bjsm.2009.066837.

27.Pontillo M, Orishimo KF, Kremenic IJ, McHugh MP, Mullaney MJ, Tyler TF. Shoulder musculature activity and stabilization during upper extremity weight-bearing activities. N Am J Sports Phys Ther. 2007;2(2):90-96.

28. Flegal KM, Kit BK, Graubard BI. Body mass index categories in observational studies of weight and risk of death. Am J Epidemiol. 2014;180(3):288-296. doi:https://doi.org/10.1093/aje/kwu111

29. Ekstrom RA, Bifulco KM, Lopau CJ, Andersen CF, Gough JR. Comparing the function of the upper and lower parts of the serratus anterior muscle using surface electromyography. J Orthop Sports Phys Ther. 2004;34(5):235-243. doi: 10.2519/jospt.2004.34.5.235.

30. Bailey LB, Beattie PF, Shanley E, Seitz AL, Thigpen CA. Current rehabilitation applications for shoulder ultrasound imaging. J Orthop Sports Phys Ther. 2015;45(5):394-405. doi: 10.2519/jospt.2015.4232.

31 Huang Q, Li D, Zhang Y, Hu A, Huo M, Maruyama H. The Reliability of Rehabilitative Ultrasound Imaging of the Cross-sectional Area of the Lumbar Multifidus Muscles in the PNF Pattern. J Phys Ther Sci. 2014;26(10):1539-1541. doi:10.1589/jpts.26.1539.

32. Gorgey AS, Timmons MK, Michener LA, Ericksen JJ, Gater DR. Intrarater reliability of ultrasound imaging of wrist extensor muscles in patients with tetraplegia. PM R. 2014;6(2):127-133. doi: 10.1016/j. pmrj.2013.08.607.

33. McGaugh J, Ellison J. Intrasession and interrater reliability of rehabilitative ultrasound imaging measures of the deep neck flexors: A pilot study. Physiother Theory Pract. 2011;27(8):572-577. doi: 10.3109/09593985.2010.544706.

34. Seitz AL, Kocher JH, Uhl TL. Immediate effects and short-term retention of multi-modal instruction compared to written only on muscle activity during the prone horizontal abduction exercise in individuals with shoulder pain. J Electromyogr Kinesiol. 2014;24(5):666-674. doi: 10.1016/j.jelekin.2014.05.006.

35. Escamilla RF, Yamashiro K, Paulos L, Andrews JR. Shoulder muscle activity and function in common shoulder rehabilitation exercises. Sports Med. 2009;39(8):663-685. doi: 10.2165/00007256200939080-00004.

36. Potluri S, Lampa SJ, Norton AS, Laskowski MB. Morphometric analysis of neuromuscular topography in the serratus anterior muscle. Muscle Nerve. 2006;33(3):398-408. doi:10.1002/mus.20470.

37. Szucs K, Navalgund A, Borstad JD. Scapular muscle activation and coactivation following a fatigue task. Med Biol Eng Comput. 2009;47(5): 487-495. doi: 10.1007/s11517-009-0485-5. 